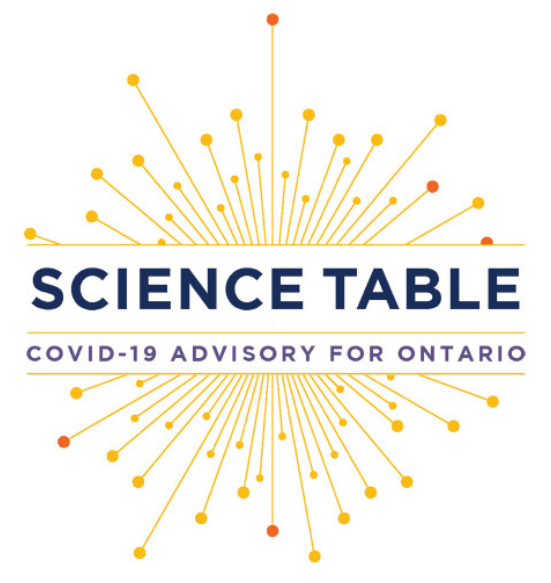

Version: $\mathbf{1 . 0}$

Published: July 21, 2021

Citation: Katz GM, Born KB, de Wit M, et al. COVID-19 vaccine certificates: key considerations for the Ontario context. Science Briefs of the Ontario COVID-19 Science Advisory Table. 2021;2(39). https:// doi.org/10.47326/ocsat.2021.02.39.1.0

Author Affiliations: The affiliations of the members of the Ontario COVID-19 Science Advisory Table can be found at https:// covid19-sciencetable.ca/.

Declarations of Interest: The declarations of interest of the members of the Ontario COVID-19 Science Advisory Table, its Working Groups, or its partners can be found at https:// covid19-sciencetable.ca/. The declarations of interest of external authors can be found under additional resources at https://doi. org/10.47326/ocsat.2021.02.39.1.0

About Us: The Ontario COVID-19 Science Advisory Table is a group of scientific experts and health system leaders who evaluate and report on emerging evidence relevant to the COVID-19 pandemic, to inform Ontario's response. Our mandate is to provide weekly summaries of relevant scientific evidence for the COVID-19 Health Coordination Table of the Province of Ontario, integrating information from existing scientific tables, Ontario's universities and agencies, and the best global evidence. The Science Table summarizes its findings for the Health Coordination Table and the public in Science Briefs.

Correspondence to: Secretariat of the Ontario COVID-19 Science Advisory Table (info@covid19-sciencetable.ca)

Copyright: 2021 Ontario COVID-19 Science Advisory Table. This is an open access document distributed under the terms of the Creative Commons Attribution License, which permits unrestricted use, distribution, and reproduction in any medium, provided that the original work is properly cited.

The views and findings expressed in this Science Brief are those of the authors and do not necessarily reflect the views of all of the members of the Ontario COVID-19 Science Advisory Table, its Working Groups, and its partners.
SCIENCE BRIEFS

\section{COVID-19 Vaccine Certificates: Key Considerations for the Ontario Context}

Gabrielle M. Katz, Karen B. Born, Melanie de Wit, Kwame McKenzie, Colleen M. Flood, Cameron Bell, Charles Cooper-Simpson, Gerald A. Evans, Jennifer L. Gibson, Jessica Hopkins, Akwatu Khenti, Yona Lunsky, Antonina Maltsev, Allison McGeer, Andrew M. Morris, Menaka Pai, Anna Perkhun, Fahad Razak, Robert J. Reid, Paula A. Rochon, Beate Sander, Michael Schull, Brian Schwartz, Arthur S. Slutsky, Maxwell J. Smith, Robert Steiner, Tania Watts, Kumanan Wilson, Peter Jüni, Nathan M. Stall on behalf of the Ontario COVID-19 Science Advisory Table

\section{Key Message}

Many jurisdictions are developing and implementing COVID-19 vaccine certificates as falsification-proof, verifiable proof of immunization in secure digital or paperbased formats.

Vaccine certificates can be used to regulate entry into discretionary settings that pose a high risk for SARS-CoV-2 transmission (e.g., indoor dining, bars, gyms, cultural and sports events).

Vaccine certificates can also be used in non-discretionary settings (e.g., schools, universities, congregate settings, and workplaces), especially in settings that require mandatory vaccination.

On a short-term basis, vaccine certificates could enable the re-opening of high-risk settings sooner and/or at increased capacity. Vaccine certificates will be of particular importance to maintain economic and societal reopening if public health measures need to be reintroduced. Some jurisdictions are also implementing vaccine certificates with the goal of incentivizing COVID-19 vaccination.

On a longer-term basis, vaccine certificates can serve as a verifiable, secure, standardized, accessible and portable records of immunization.

There is currently no scientific evidence of the direct impact of COVID-19 vaccine certificates on SARS-CoV-2 transmission or population vaccination rates, and there are important ethical, legal, accessibility, and privacy considerations concerning their development and implementation.

\section{Summary}

\section{Background}

A COVID-19 vaccine certificate is a verifiable attestation by an issuing body that a person received an approved and complete series of COVID-19 vaccines. These are distinct from vaccine passports which provide proof of vaccination status for the purpose of travel across international borders and require multinational consensus. COVID-19 vaccine certificates are being developed and used in many countries and jurisdictions with varying eligibility requirements and intended purposes. Examining the development, implementation, and uses of COVID-19 vaccine certificates across different jurisdictions can help inform whether vaccinate certificates have a role in Ontario's reopening as well as longer term uses as a record of immunization.

\section{Questions}

How are vaccine certificates being developed, and what are the key design 
considerations?

What are the main use cases for COVID-19 vaccine certificates?

Which jurisdictions are using vaccine certificates, and who is considered eligible to hold one?

What are settings in which vaccine certificates are currently being used?

What is the scientific and public health evidence supporting the use of vaccine certificates in incentivizing COVID-19 vaccination and reducing SARS-CoV-2 transmission?

What are the legal, ethical, equity, privacy, and accessibility concerns associated with vaccine certificates, and can they be resolved?

\section{Findings}

Globally, many jurisdictions are developing and implementing digital and paperbased COVID-19 vaccine certificates, and several multinational certificate programs are underway.

Beyond verification of vaccination in a patient's health record, vaccine certificates can be used to regulate entry into discretionary settings that pose a high risk for SARS-CoV-2 transmission (e.g., indoor dining, bars, gyms, cultural and sports events). Vaccine certificates can also be used in non-discretionary settings (e.g., schools, universities, congregate settings, and workplaces), especially in settings that require mandatory vaccination.

On a short-term basis, vaccine certificates could enable the re-opening of high-risk settings sooner and/or at increased capacity. Vaccine certificates will be of particular importance to maintain economic and societal reopening if public health measures need to be reintroduced. Some jurisdictions are also implementing vaccine certificates with the goal of incentivizing COVID-19 vaccination. On a longer-term basis, vaccine certificates can serve as a verifiable, secure, standardized, accessible, and portable records of immunization.

There is a strong scientific basis for COVID-19 vaccination reducing infection, hospitalization, and severe illness from SARS-CoV-2. Limiting participation of higherrisk activities for SARS-CoV-2 transmission to vaccinated individuals or those who can demonstrate that they are at low current risk of SARS-CoV-2 infection should theoretically reduce the risk of transmission and infection for both vaccinated and unvaccinated people. However, there is currently no direct scientific evidence of the impact of COVID-19 vaccine certificates on vaccine coverage or SARS-CoV-2 transmission.

There are also important ethical, legal, accessibility, and privacy considerations with varying impacts on different populations that should be considered in the design and implementation of COVID-19 vaccine certificates. Government and health authorities will need to take steps to ensure that COVID-19 vaccination, as well as vaccine certification, is accessible to all individuals on equitable terms, that necessary accommodations are implemented for specific groups and individuals to the extent possible, and that any incursion on privacy is limited to what is necessary to achieve the intended purpose.

Concerns that the regulation and enforcement of vaccine certificates may contribute to discrimination should be carefully considered and monitored. The evidence of the effectiveness of a COVID-19 vaccine certificate to meet particular public health needs will vary between settings. In each case the need for a COVID-19 vaccine certificates will have to be balanced against the ethical, legal, accessibility and privacy considerations. Vaccine certificates should be used only where and for as long as they have a compelling policy objective of protecting populations or individuals who are 
more at risk of contracting SARS-CoV-2.

\section{Interpretation}

COVID-19 vaccine certificates may have a practical short-term utility for supporting and maintaining economic and societal reopening. In the longer-term, vaccine certificates may be useful as verifiable, secure, standardized, and accessible electronic or paper records of immunization. Further, given the anticipated seasonality of SARS-CoV-2 as well as the possibility of additional variants of concern, establishing the infrastructure for COVID-19 vaccine certification may be useful if public health measures need to be reintroduced during future potential waves.

Governments and health authorities have a role in ensuring that necessary regulations are in place to ensure that vaccine certificates are created and used in a way that safeguards human rights, enables individual accommodations when warranted (e.g., legitimate medical exemptions), and protect against misuse. Provincial and territorial governments may want to consider a common design of vaccine certificates that reflects key development and implementation principles. There are concerns that in the absence of government regulation or direction, organizations and sectors will create their own systems. Governments also have a role in defining the settings where vaccine certificate requirements for those accessing services are prohibited (e.g., health care, essential services).

\section{Background}

The current COVID-19 vaccination campaign is one of the largest mass immunization efforts in modern history. Verification of COVID-19 vaccination status is important as individuals return to work and school, international travel resumes, and society and the economy fully reopen. ${ }^{1}$ Verified proof of immunization in the form of COVID-19 vaccine certificates are being developed and used in multiple settings across the world with varying eligibility requirements and intended purposes.

A COVID-19 vaccine certificate is a verifiable attestation by an issuing body, for example, the Province of Ontario, that a person received an approved and complete series of COVID-19 vaccines. It is important to differentiate vaccine certificates from vaccine passports, certificates of recovery, and immunity certificates, especially since the terms are often used interchangeably but have very different meanings and potential uses.

A vaccine passport provides proof of vaccination status for the purpose of travel across international borders and requires multinational consensus. A certificate of recovery signifies that a person has been infected with SARS-CoV-2 and recovered from COVID-19, using SARS-CoV-2 test results or alternative methods of measuring immunity. An immunity certificate combines elements of vaccine certificates and certificates of recovery, confirming that an individual has a certain level of immunity to SARS-CoV-2, whether from vaccination or previous infection. ${ }^{2,3}$

As the federal government has already signaled, a Canadian COVID-19 vaccine passport will be developed and implemented nationally and will be distinct from COVID-19 vaccine certificates which would be the responsibility of individual provinces and territories as the entities responsible for vaccinating their residents. ${ }^{4}$ There are technical challenges related to the collection, storage, and sharing of vaccine documentation across Canadian provinces and territories. ${ }^{5}$ Vaccine certificate programs are under consideration in several Canadian provinces and territories including British Columbia, New Brunswick, Northwest Territories, and Nova Scotia. ${ }^{5}$ On July 8, 2021, Quebec announced that digital proof of vaccination may be required to enter moderate or high risk settings for COVID-19 transmission. Settings such as gyms, team sports, and theatres may require proof of vaccination in the event of rising cases 
of COVID-19 once the entire eligible population has vaccine access. ${ }^{6}$ Manitoba has introduced digital or paper-based "immunization cards" that can be used for domestic travel without quarantine, expanded visitation permission in hospitals and long-term care (LTC) homes, and indoor dining with individuals outside of one's household. 7,8

As Ontario continues with the mass COVID-19 vaccination campaign and moves through its three-step reopening plan guided by health indicators and province-wide COVID-19 vaccination rates, ${ }^{9}$ it is helpful to examine how vaccine certificates are being developed and implemented globally. Examining how vaccine certificates are being used across different jurisdictions globally may help inform a potential role in Ontario. Survey response: $₫$ No response/do not know $₫$ Oppose vaccine passports $₫$ Neither $₫$ Support vaccine passports

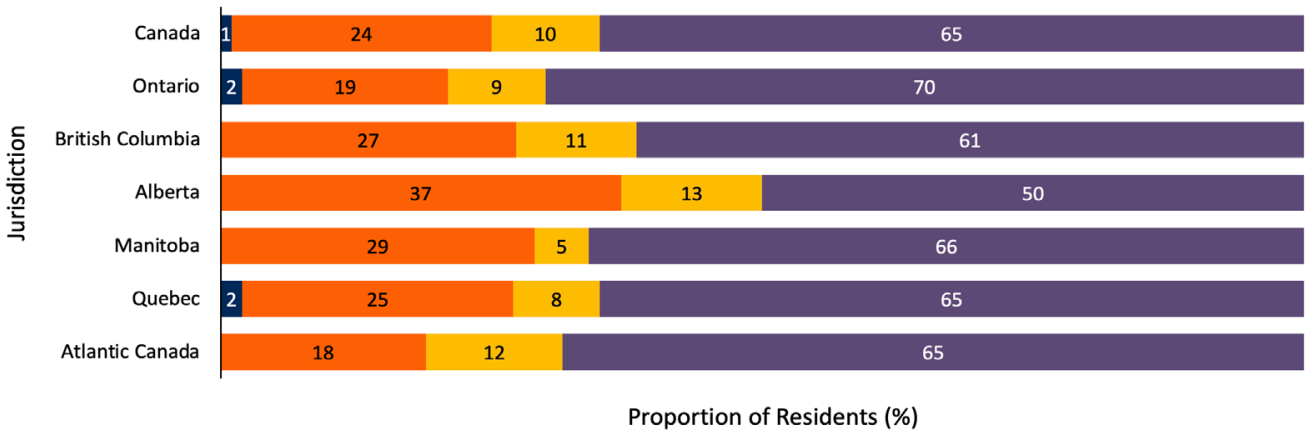

Figure 1. Support and Opposition Towards Vaccine Passport Implementation in Canada

Graph demonstrating the percentage, in a random sample of 1,115 adult Canadian residents, who support or oppose the implementation of vaccine passports for travel or certain discretionary activities. Participants were asked to rank their support on a scale of 1 to 5, where 1 and 2 is "oppose", 3 is "neither", and 4 and 5 is "support." Atlantic Canada includes the provinces of New Brunswick, Newfoundland and Labrador, and Prince Edward Island. The survey took place from May 28 to June 3, 2021. Data sourced from personal correspondence with Frank Graves.

In a recent survey, 1,115 adult Canadian residents were asked, "to what extent would you support or oppose a proof-of-vaccination system where Canadians would be required to carry a vaccine passport in order to travel or attend public events like concerts and sporting events?" A substantial proportion of respondents showed support. A total of $65 \%$ of all respondents and $70 \%$ of respondents from Ontario supported the use of a proof-of-vaccination system for travel or certain discretionary activities.

The survey results are congruent to other market research surveys that have been conducted by Ipsos and the Angus Reid Institute. ${ }^{10,11}$ These survey data are statistically weighted by age, gender, and region. However, due to limitations on sample size and issues accessing respondents from marginalized and underrepresented groups, these surveys are likely not an accurate representation of all Canadian residents' sentiments.

\section{Questions}

How are vaccine certificates being developed, and what are the key design considerations?

What are the main use cases for COVID-19 vaccine certificates?

Which jurisdictions are using vaccine certificates, and who is considered eligible to hold one?

What are settings in which vaccine certificates are currently being used?

What is the scientific and public health evidence supporting the use of vaccine certificates in incentivizing COVID-19 vaccination and reducing SARS-CoV-2 transmission?

What are the legal, ethical, equity, privacy and accessibility concerns associated with vaccine certificates, and can they be resolved? 


\section{Findings}

\section{COVID-19 Vaccine Certificate Development and Key Design Principles}

The European Commission has outlined three elements of any COVID-19 vaccine certificate: a minimum dataset, a standard unique identifier, and a trust framework. ${ }^{12}$ A minimum dataset includes verification that: 1) the certificate has been issued by an authorized entity, 2) the information presented on the certificate is authentic, valid, and has not been altered, and 3) the certificate can be linked to the holder of the certificate. The standard unique identifier, a numeric or alphanumeric string that is associated with a single entity within a given system, can be used as a link to the underlying data registry using either a digital or paper-based platforms. The trust framework provides the basis for establishing the vaccine certificates' authenticity, integrity, and validity by defining the rules, policies, protocols, formats, and standards needed.

The World Health Organization (WHO) has proposed several key design principles for vaccine certificates. ${ }^{13}$ The WHO's "Interim guidance for developing a Smart Vaccination Certificate" proposes that the design of any certificate must be equitable (everyone has the right to obtain and hold a vaccine certificate, and the certificate should not exacerbate existing health inequities or increase the digital divide), accessible (vaccine certificates should function in online and offline environments across multiple platforms), and protect privacy (retain the certificate holder's control of personal health information and collect only necessary data). The guidance states vaccine certificates should also be adaptable, flexible, and environmentally sustainable. ${ }^{13}$

Vaccine certificates can either be digital (available as a scannable barcode or Quick Response (QR) code that is either stored on a smartphone application or printed) or paper-based. ${ }^{13,14}$ In the digital form, vaccine certificates allow individuals to present documentation of vaccination which can be cryptographically verified by interested parties. ${ }^{13}$ Cryptographic verification involves generating a secure and unique digital signature that can be used by interested parties to verify that an individual's immunization record was issued by a trusted organization. There are some equity and accessibility benefits to traditional paper-only vaccination certifications. However, paper vaccine certificates may be more susceptible to fraud, forgery, and more easily lost and damaged. ${ }^{13,15}$

Typically, vaccine certificates are scanned prior to entry into places or activities that requires proof of immunization. ${ }^{14}$ In Israel, individuals holding a "Green Pass" (vaccine certificate) were required to provide additional proof of identity with governmentissued photo identification that matched the name on the vaccine certificate. ${ }^{14,16}$

\section{Principal Use Cases for COVID-19 Vaccine Certificates}

Governments, public health officials, and institutions may consider certain places or events that are high risk for infection and transmission of SARS-CoV-2. People attending such events or places may need to demonstrate proof that they are at low risk of having COVID-19 as a means of protecting populations or individuals who are more at risk of contracting SARS-CoV-2. ${ }^{17}$ Vaccine certificates have been considered one practical way of identifying one group of low-risk individuals. The major use cases for COVID-19 vaccine certificates (the main scenarios in which they could be used) proposed in the literature and being used in several jurisdictions across the world are entry to settings that pose a high risk for SARS-CoV-2 transmission for discretionary activities or non-discretionary activities.

Settings regarded as having a high risk for SARS-CoV-2 transmission occur under three main conditions, often described as "the $3 \mathrm{C}^{\prime} \mathrm{s}$ ": closed settings with poor ventilation, crowded places with many people nearby, and close-contact settings. ${ }^{18-20}$ 
In settings for discretionary activities with a higher risk of SARS-CoV-2 transmission, vaccine certificates could be used as proof of immunization to gain entry. This would include settings such as large sporting and cultural events, indoor dining, gyms, hotels, etc. Examples of COVID-19 vaccine certificates being used for these purposes include Israel's Green Pass, Denmark's Corona passport, and New York State's Excelsior Pass (see Tables 1 and 2).

In settings for non-discretionary activities, vaccine certificates could be used in places such as schools, congregate settings, universities, and other workplaces (such as health care) where proof of COVID-19 vaccination would be required for employment and/ or enrollment. For example, several Ontario universities (including Western University and the University of Toronto) have announced that they will require students living in residence to be fully vaccinated against COVID-19 by Fall 2021. ${ }^{21-23}$ Students required to show proof of vaccination could use a verifiable and secure vaccine certificate to do so. As well, Ontario has mandated a COVID-19 immunization policy for LTC staff. Staff are required to do one of the following: provide proof of vaccination; provide a documented medical reason for not getting vaccinated, or participate in an educational program about COVID-19 vaccination. ${ }^{24}$

There are essential and low-risk settings that should not be included in use cases of COVID-19 vaccine certificates. Vaccination status should not prevent individuals from accessing health services, grocery stores or other essential services, or enter settings which are considered to have a low risk of SARS-CoV-2 transmission such as uncrowded outdoor spaces. Regulating the use of vaccine certificates to these essential settings ensures that unvaccinated individuals are protected but not unduly discriminated against.

The Government of Canada recently announced plans to lift quarantine requirements for returning travelers who are Canadian citizens or permanent residents and have received a complete series of approved COVID-19 vaccines. These travelers must take a SARS-CoV-2 test before leaving and upon arrival to Canada, but there is no required quarantine period while awaiting test results. These policy changes will be implemented on July 5, 2021.25,26

Prior to their arrival, inbound Canadian travelers must upload their documentation of vaccination status, recent travel history, and results of a SARS-CoV-2 test taken within 72 hours to the ArriveCAN software application or the government website. The federal government also announced that a national vaccine passport is being developed; however, temporary measures to confirm vaccination status will need to be in place by early July since vaccine passports are not expected to be ready by then. ${ }^{2}$

\section{Eligibility Criteria and Implementation Statuses of 'COVID-19 Certificates'}

Table 1 summarizes four international 'COVID-19 certificates' - hereafter used to describe proof of vaccination, recovery, or recent negative test results - and their implementation statuses, as well as respective eligibility requirements and steps for certificate retrieval and presentation. The COVID-19 certificates described in Table 1 all function as verifiable proof of vaccination (vaccine certificates) for eligible individuals. All are available in digital and paper-based formats, with security features to prevent falsification. Some are country-specific (e.g., Israel); however, others are multinational (e.g., European Union (EU) Digital COVID Certificate). 


\begin{tabular}{|c|c|c|c|}
\hline Jurisdiction & $\begin{array}{c}\text { Name, Certificate Type, } \\
\text { and Implementation } \\
\text { Status }\end{array}$ & Eligibility Criteria & $\begin{array}{l}\text { Certificate Acquisition } \\
\text { and Presentation }\end{array}$ \\
\hline Israel & $\begin{array}{l}\text { Green Pass }^{27} \\
\text { COVID-19 vaccine certificate; } \\
\text { can also function as a certificate } \\
\text { of recovery or certificate of a } \\
\text { recent negative test. } \\
\text { Introduced on February 21, } \\
2021 \text {. Green Pass requirements } \\
\text { expired on June } 1,2021 \text {. }\end{array}$ & $\begin{array}{l}\text { Individuals who were fully } \\
\text { vaccinated with a complete } \\
\text { series of approved COVID-19 } \\
\text { vaccines. } \\
\text { Additional criteria: } \\
\text { - Individuals who were con- } \\
\text { sidered recovered from } \\
\text { COVID-19. }{ }^{28} \\
\text { Unvaccinated individuals } \\
\text { aged } 16 \text { years and younger } \\
\text { who received a negative } \\
\text { SARS-CoV-2 PCR test result } \\
\text { could obtain a Green Pass } \\
\text { that was valid for } 72 \text { hours } \\
\text { from their day of testing. }\end{array}$ & $\begin{array}{l}\text { - Options to apply online, } \\
\text { on a smartphone app, or } \\
\text { by phone. } \\
\text { - Green Pass could be pre- } \\
\text { sented as a QR code on } \\
\text { a Ministry of Health App } \\
\text { or using a printed copy, } \\
\text { along with matching } \\
\text { photo ID. }\end{array}$ \\
\hline $\begin{array}{l}\text { European Union } \\
\text { (EU), Iceland, } \\
\text { Switzerland, } \\
\text { Liechtenstein, } \\
\text { and Norway }\end{array}$ & $\begin{array}{l}\text { EU Digital COVID Certificate }{ }^{29,30} \\
\text { COVID-19 vaccine certificate; } \\
\text { can also function as a certificate } \\
\text { of recovery or certificate of a } \\
\text { recent negative test, and as a } \\
\text { recovery passport or recent } \\
\text { negative test passport. } \\
\text { National authorities (e.g., test } \\
\text { centres, health authorities, } \\
\text { and eHealth portals) are } \\
\text { responsible for issuing the } \\
\text { certificate, following } \\
\text { requirements, to create and } \\
\text { implement their own versions } \\
\text { of the digital certificate which } \\
\text { can be accepted by other } \\
\text { Member States. } \\
\text { Certificates are currently } \\
\text { being issued in Bulgaria, Czech } \\
\text { Republic, Denmark, Germany, } \\
\text { Greece, Croatia and Poland. } \\
\text { The certificate will become } \\
\text { available in the rest of the EU, } \\
\text { Iceland, Norway, Switzerland, } \\
\text { and Liechtenstein as of July } 1 \text {, } \\
2021 \text {. The regulation will apply } \\
\text { for at least } 12 \text { months. }\end{array}$ & $\begin{array}{l}\text { Individuals who are vaccinated } \\
\text { against COVID-19. Member } \\
\text { States can decide if they accept } \\
\text { certificates after one or two } \\
\text { doses of a COVID-19 vaccine. } \\
\text { Additional criteria: } \\
\text { - Individuals who have re- } \\
\text { ceived a negative SARS- } \\
\text { CoV-2 test result; accept- } \\
\text { able tests are specified } \\
\text { by each Member State. } \\
\text { Maximum certificate valid- } \\
\text { ity time is } 72 \text { hours for PCR } \\
\text { tests and - when accepted } \\
\text { by a Member State - } 48 \\
\text { hours for rapid antigen } \\
\text { tests. } \\
\text { Individuals who are con- } \\
\text { firmed to have recovered } \\
\text { from SARS-CoV-2 infection } \\
\text { following a positive PCR } \\
\text { or rapid antigen test. Max- } \\
\text { imum certificate validity } \\
\text { period for recovered indi- } \\
\text { viduals is } 180 \text { days. }\end{array}$ & $\begin{array}{l}\text { - Member States agreed } \\
\text { on a common design } \\
\text { for electronic and paper } \\
\text { versions of the certifi- } \\
\text { cate. Both will contain a } \\
\text { QR code with essential } \\
\text { information and a digital } \\
\text { signature to prevent fal- } \\
\text { sification. } \\
\text { Certificates contain only } \\
\text { necessary information, } \\
\text { including name, date of } \\
\text { birth, certificate issu- } \\
\text { er, a unique identifier, } \\
\text { and a minimum set of } \\
\text { information about an } \\
\text { individual's vaccination, } \\
\text { testing, or recovery. }\end{array}$ \\
\hline Denmark & $\begin{array}{l}\text { Corona Passport (Coronapas) } \\
\text { COVID-19 vaccine certificate; } \\
\text { can also function as a certificate } \\
\text { of recovery or certificate of a } \\
\text { recent negative test, and as a } \\
\text { recovery passport or recent } \\
\text { negative test passport. } \\
\text { Corona passport requirements } \\
\text { were introduced on March } \\
22 \text {, } 2021 \text {. The passport was } \\
\text { developed to meet the EU } \\
\text { requirements specified for the } \\
\text { EU Digital COVID Certificate. }\end{array}$ & $\begin{array}{l}\text { Individuals may receive a valid } \\
\text { corona passport } 14 \text { to } 42 \text { days } \\
\text { after the date of their first } \\
\text { COVID-19 vaccine and can } \\
\text { receive a valid corona passport } \\
\text { immediately after they have } \\
\text { been fully vaccinated. The } \\
\text { corona passport is valid for } \\
\text { eight months after being fully } \\
\text { vaccinated. } \\
\text { Additional criteria: } \\
\text { - Individuals who have re- } \\
\text { ceived a negative test result } \\
\text { (PCR or rapid antigen test). } \\
\text { The corona passport is valid } \\
\text { for } 72 \text { hours from the time } \\
\text { of the test. } \\
\text { Individuals who have been } \\
\text { infected with COVID-19 and } \\
\text { have a positive PCR test re- } \\
\text { sult within the past } 14 \text { to } \\
180 \text { days. }\end{array}$ & $\begin{array}{l}\text { - The corona passport is } \\
\text { available as a scannable } \\
\text { QR code through several } \\
\text { apps. Non-digital ver- } \\
\text { sions are available. } \\
\text { - If using the corona } \\
\text { passport app for pass } \\
\text { presentation, ID is not } \\
\text { required. Individuals } \\
\text { can choose whether to } \\
\text { display their name and } \\
\text { date of birth using the } \\
\text { app. }\end{array}$ \\
\hline
\end{tabular}




\begin{tabular}{|c|c|c|c|}
\hline & & $\begin{array}{l}\text { Exemptions made for chil- } \\
\text { dren aged } 15 \text { years and } \\
\text { below and those who, due } \\
\text { to medical reasons or a dis- } \\
\text { ability, should not have a } \\
\text { COVID-19 test. }\end{array}$ & \\
\hline New York State & $\begin{array}{l}\text { Excelsior Pass }^{32,33} \\
\text { COVID-19 vaccine certificate; } \\
\text { can also function as a certificate } \\
\text { of a recent negative test. } \\
\text { Introduced on March 26, } 2021 .\end{array}$ & $\begin{array}{l}\text { Fully vaccinated individuals, } \\
15 \text { days after their final dose } \\
\text { of COVID-19 vaccine (valid for } \\
365 \text { days). } \\
\text { Additional criteria: } \\
\text { - Individuals who receive a } \\
\text { negative COVID-19 PCR test } \\
\text { result (valid for } 3 \text { days after } \\
\text { the time of the test). } \\
\text { - Individuals who receive a } \\
\text { negative COVID-19 antigen } \\
\text { test result (valid for } 6 \text { hours } \\
\text { after the time of the test). } \\
\text { - All individuals must not } \\
\text { have tested positive for } \\
\text { COVID-19 in the previous } \\
10 \text { days. }\end{array}$ & $\begin{array}{l}\text { - Passes are obtained by } \\
\text { following the instruc- } \\
\text { tions on the Excelsior } \\
\text { Pass Wallet App or the } \\
\text { Excelsior Pass website. } \\
\text { From the website, print- } \\
\text { ed passes and screen- } \\
\text { shots can also be re- } \\
\text { trieved. } \\
\text { - The pass's QR code is } \\
\text { scanned using the Ex- } \\
\text { celsior Pass Scanner } \\
\text { app; photo ID must be } \\
\text { presented alongside the } \\
\text { pass. }\end{array}$ \\
\hline
\end{tabular}

Table 1. Jurisdictional Scan of Vaccine Certificate Eligibility and Implementation

Table presenting the implementation status, involved jurisdictions, eligibility criteria, and certificate application and presentation processes for four international COVID-19 vaccine certificates. QR, quick response.

The COVID-19 certificates shown in Table 1 were chosen to represent the wide range of vaccine certificates being implemented globally - with regional, national, and multinational examples, and varying uses (see Table 2).

The COVID-19 certificates described have several aspects in common: they are available in multiple formats (digital, paper-based); there are efforts made to prevent falsification, and only necessary health information is collected from certificate holders. Furthermore, there are factors specific to certain jurisdictions that improve the efficiency of implementation of the COVID-19 certificates. Multinational collaboration was essential in the planning and implementation of the EU Digital COVID Certificate as these certificates - issued nationally by each Member State - are accepted at several international borders. Leveraging existing technologies was also useful; for example, Israel's centralized digital infrastructure was vital to the implementation of the Green Pass. ${ }^{16}$

\section{Current Global Use of 'COVID-19 Certificates'}

Table 2 describes the domestic settings in which these four COVID-19 certificates can be used.

\begin{tabular}{|c|c|}
\hline Name & Usage in Domestic Settings \\
\hline $\begin{array}{l}\text { Green Pass } \\
\text { (Israel) }\end{array}$ & $\begin{array}{l}\text { - Green Pass holders were granted access to sport and cultural event venues and } \\
\text { concert halls. All public health measures and Green Pass requirements, excluding } \\
\text { indoor masking, were removed on June 1, } 2021 \text {. } \\
\text { - In earlier phases of reopening in February and March 2021, restaurants, gyms, } \\
\text { hotels, pools, and sports facilities also had Green Pass requirements, and public } \\
\text { health measures such as Purple Badge requirements guided the safe reopening } \\
\text { of non-essential workplaces. }{ }^{16}\end{array}$ \\
\hline $\begin{array}{l}\text { EU Digital COVID } \\
\text { Certificate }{ }^{30,37} \\
\text { (European Union (EU), } \\
\text { Iceland, Switzerland, Liech- } \\
\text { tenstein, and Norway) }\end{array}$ & $\begin{array}{l}\text { - National authorities are responsible for issuing vaccine certificates and can thus } \\
\text { introduce vaccine certificate requirements in domestic settings (e.g., Denmark } \\
\text { - see below). } \\
\text { - Additional use: Digital COVID certificates could facilitate free movement for trav- } \\
\text { el between Member States, unless restrictions are necessary for public health } \\
\text { purposes. }\end{array}$ \\
\hline
\end{tabular}




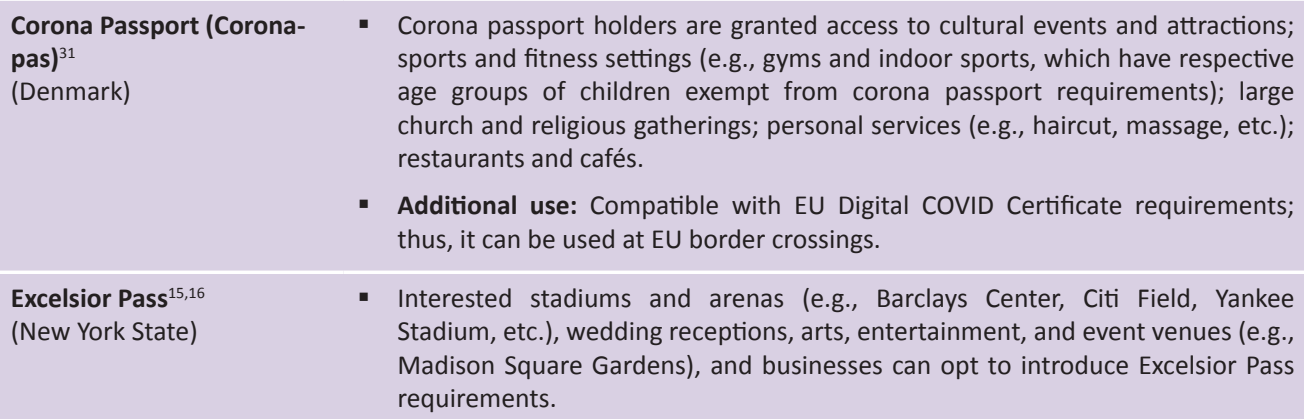

Excelsior Pass ${ }^{15,1}$ (New York State)

- Corona passport holders are granted access to cultural events and attractions; sports and fitness settings (e.g., gyms and indoor sports, which have respective age groups of children exempt from corona passport requirements); large church and religious gatherings; personal services (e.g., haircut, massage, etc.); restaurants and cafés.

- Additional use: Compatible with EU Digital COVID Certificate requirements; thus, it can be used at EU border crossings.

- Interested stadiums and arenas (e.g., Barclays Center, Citi Field, Yankee Stadium, etc.), wedding receptions, arts, entertainment, and event venues (e.g., Madison Square Gardens), and businesses can opt to introduce Excelsior Pass requirements.

Table 2. Jurisdictional COVID-19 Certificate Requirements in Domestic Settings

Table presenting the domestic settings in which COVID-19 certificates are in use for four international vaccine certificates.

The COVID-19 certificates detailed in Tables 1 and 2 may be used for entry to restricted places and activities, many of which are high-risk settings for the transmission of SARSCoV-2 (close contact, crowded, or closed spaces). Beyond the COVID-19 certificates mentioned, proof of vaccination - in digital form or otherwise - may be required in other settings, for example, in university residences, as a condition of employment in LTC homes or other healthcare settings. Thus, the intention behind these certificates varies, with some jurisdictions placing emphasis on protecting high-risk individuals and reducing SARS-CoV-2 transmission, ${ }^{16}$ and others implementing them with the goal of incentivizing COVID-19 vaccination.

\section{Scientific, Public Health and Pragmatic Considerations of Vaccine Certificates}

There is a strong scientific basis for COVID-19 vaccines reducing infection, hospitalization, and severe illness from SARS-CoV-2. ${ }^{38}$ Limiting participation of higherrisk activities for COVID-19 transmission to vaccinated individuals should theoretically reduce the risk of SARS-CoV-2 infection and transmission. However, there is no direct evidence of the impact vaccine certificates might have on the spread of the SARSCoV-2 virus. ${ }^{7}$ Further evidence is required to scientifically justify the effectiveness of vaccine certificates as a policy intervention. ${ }^{5}$

In France, vaccine certificates, referred to as "health passports", will be required for individuals to access discretionary settings including restaurants and tourist attractions. ${ }^{39}$ The policy will be implemented in August 2021, and those who are fully vaccinated against COVID-19, recently recovered from SARS-CoV-2, or had a recent negative test are eligible to apply for a health passport. Healthcare workers will also be required to be vaccinated by September 15, 2021.

First dose COVID-19 vaccination booking numbers surged in anticipation of and following the French President's announcement of health passports on July 12, 2021. This data is summarized in Figure 2. 


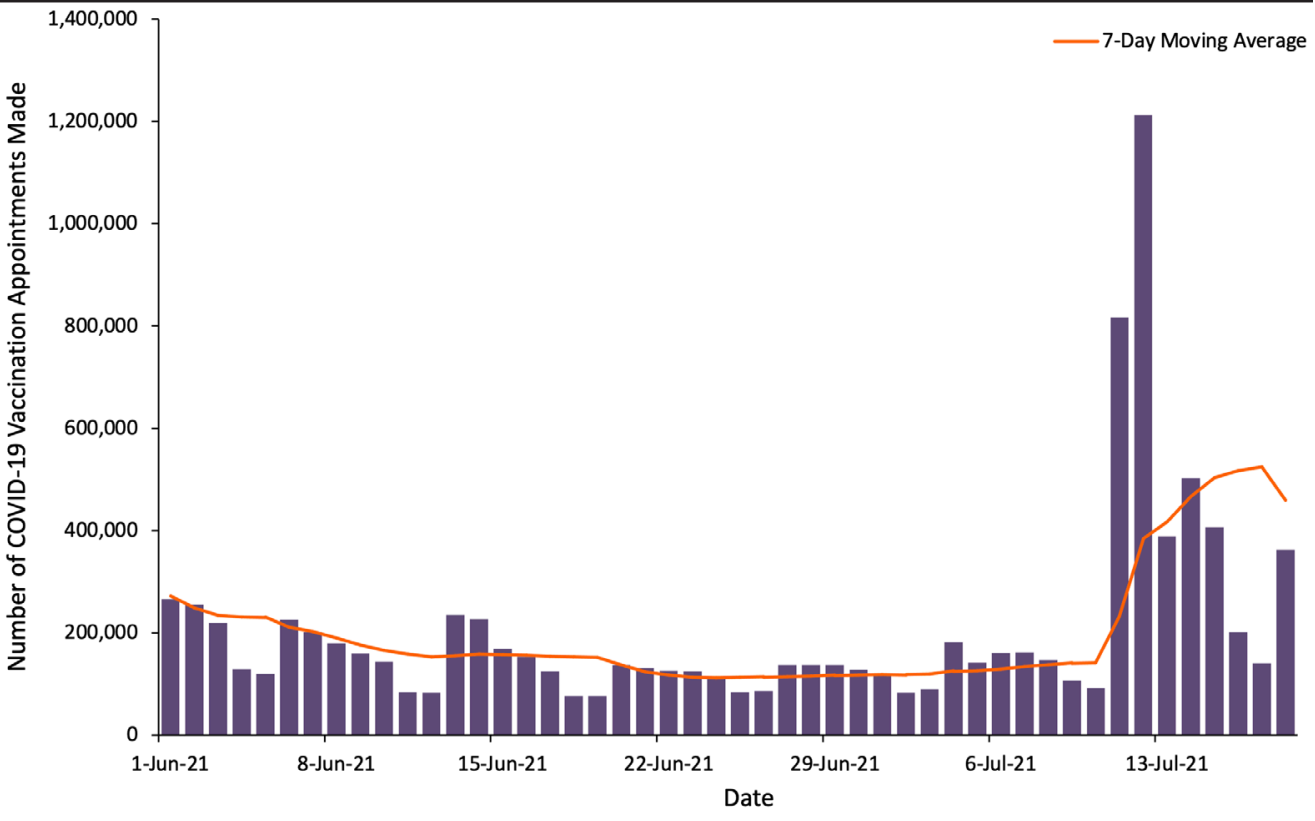

Figure 2. COVID-19 Vaccination Appointments Made per Day in France

Figure showing the number of first dose COVID-19 vaccination appointments booked using the main booking platform from June 1, 2021, to July 18, 2021. The purple bars show the daily number of appointments made, and the orange line displays the 7-day moving average. Data sourced from Doctolib, the main vaccination booking platform. ${ }^{40}$

The health passports announcement corresponded with a subsequent spike in first dose COVID-19 vaccination bookings. ${ }^{41}$ Between the day preceding the announcement and one week afterwards, approximately 3.5 million first dose appointments were booked. ${ }^{40,42}$ Those who used the main booking platform in this time period make up approximately $11 \%$ of France's unvaccinated population on July 12, 2021, including children below 12 years of age who are not yet eligible for vaccination. ${ }^{40,43}$

There are a growing number of studies demonstrating high effectiveness of COVID-19 vaccines in both reducing SARS-CoV-2 infection and preventing transmission. ${ }^{38}$ In the United States, the Centers for Disease Control and Prevention (CDC) released public guidance that fully vaccinated individuals can safely resume pre-pandemic activities in high transmission settings. ${ }^{44}$ The Public Health Agency of Canada (PHAC) released guidance on June 25, 2021 indicating that small groups of fully vaccinated individuals can interact with other fully vaccinated individuals in most outdoor and some indoor (e.g., private home) settings without needing to consistently mask and physically distance. ${ }^{45}$ There are a number of unknowns, including the duration of protection offered by COVID-19 vaccines as well as their effectiveness against variants of SARSCoV-2. This will be better understood through ongoing follow up with clinical trial participants and observational studies.

Israel's experience is instructive as the country had a rapid vaccination campaign alongside reopening of schools and businesses during the months of February and March 2021, without seeing an increase in SARS-CoV-2 cases or COVID-19 hospitalizations and deaths. The country introduced a 'Green Pass' or vaccination certificate which was in place from February 21 to June 1, 2021, alongside other nonpharmaceutical interventions to control the spread of SARS-CoV-2. The Green Pass allowed fully vaccinated or COVID-19 recovered individuals to enter higher SARS-CoV-2 transmission risk discretionary settings such as restaurants, hotels, gyms and other high risk indoor settings. ${ }^{16}$ The Green Pass had a number of goals including protecting vulnerable individuals in higher transmission-risk settings, incentivizing vaccination and allowing for society and the economy to reopen. ${ }^{46}$ Green Pass requirements expired on June 1, 2021, with decision makers noting it was no longer needed given Israel's low SARS-CoV-2 case numbers and high population COVID-19 vaccine uptake with $55.2 \%$ of the country's population being fully vaccinated. It is worth reiterating 
that there is no direct scientific evidence that the Green Pass was responsible for the sustained decrease in SARS-CoV-2 cases during Israel's reopening, and there are other international jurisdictions that reopened without vaccine certification, and similarly did not see a rise in cases.

\section{Legal, Ethical, Accessibility, and Privacy Considerations for COVID-19 Vaccine Certificates in Ontario}

COVID-19 vaccine certificates may be used to facilitate societal and economic reopening, and have longer-term uses as verifiable, standardized, and portable records of immunization. Fundamentally, the certificates are intended to protect those who cannot or will not be vaccinated by limiting access to settings in which the risk of SARS-CoV-2 transmission is high. ${ }^{17}$ However, there are important ethical, legal, accessibility and privacy considerations that should guide design and implementation to ensure effects are not disproportionate to the aim.

These considerations primarily relate to the potential for differential impacts of vaccine certificates on individuals and groups due to equity and accessibility considerations, discrimination concerns, and the need for privacy and security of personal health information.

Government and health authorities must take steps to ensure that COVID-19 vaccine certification is accessible to all individuals on equitable terms, and that adversely impacted populations are accommodated to the extent possible. Further, that any incursion on privacy for vaccine certificates is limited to what is necessary to achieve intended and legitimate purposes. It is also essential that vaccine certificates are used only for as long as necessary to avoid COVID-19 outbreaks, based on guidance from public health officials. For example, Israel introduced their 'Green Pass' requirements in February 2021, and these were used to gain access to large sporting, cultural venues and concert halls, before expiring on June 1, 2021 when there were low SARS-CoV-2 infection numbers and high COVID-19 vaccine coverage and it was felt this system was no longer needed. ${ }^{16}$ Israel has recently seen an increase in SARS-CoV-2 cases, and beginning July 21, 2021, access to weddings and similar events with more than 100 attendees will be limited to those who are vaccinated against COVID-19, recovered, or had a recent negative test. ${ }^{47}$ This may require the reintroduction of a COVID-19 certificate similar to the Green Pass. Realistically, with the possibility of SARS-CoV-2 becoming endemic and the potential need for vaccine booster shots in the future, ${ }^{48}$ some form of COVID-19 vaccination certificate may be intermittently used, as necessary, in some jurisdictions for the foreseeable future.

Ensuring necessary safeguards to protect rights and enable accommodations associated with COVID-19 vaccine certificates requires leadership by governments and health authorities; collaboration across provinces and territories will aid in the design of more uniform safeguards and accommodations. There are concerns that in the absence of government regulation or direction, private organizations and industry sectors will create their own systems. Governments also have a role in defining the settings where vaccine certificate requirements for those accessing services (e.g., health care, essential settings) are prohibited.

In the absence of government regulation, private sector entities may adopt and use vaccine certificates to grant access to settings such as sporting events, restaurants, and religious services. A transparent and inclusive process and regulatory framework that provides guidance and standards for appropriate use is necessary to build trust, encourage uptake and minimize unintended consequences.

\section{Equity and Access}

There are ethical concerns regarding the role of vaccine certificates in perpetuating 
existing social inequities and mistrust of government and health authorities. ${ }^{49}$ Inequities may be exacerbated through coercion and stigma for vaccine hesitant populations, those unable to be vaccinated for medical reasons, or those who have had or continue to experience less access to COVID-19 vaccines. ${ }^{50}$

Many racialized populations and other marginalized groups in Canada face substantial barriers to accessing COVID-19 vaccines and other health services. ${ }^{51,52}$ Vaccine certificates may exacerbate existing inequities regarding COVID-19 specifically, or more generally by leading to social exclusion, and reduced access to health and social services. ${ }^{53}$ In Ontario, efforts to address inequities in COVID-19 vaccine access were undertaken by targeted allocation strategies of more vaccine to high SARS-CoV-2 incidence communities. ${ }^{43}$ These communities also have higher ethnic group concentrations, more material deprivation, and a larger number of essential workers. ${ }^{44}$ Ontario's hotspot vaccination strategy worked to increase first dose of COVID-19 vaccines for residents of high SARS-CoV-2 incidence neighbourhoods, but it remains unclear whether this increased coverage for marginalized groups within these neighbourhoods equitably. ${ }^{54}$ Additional and expanded efforts should be taken to support access to vaccinations, particularly amongst historically marginalized populations, those with accessibility needs, and communities with disproportionate impacts of COVID-19.

Any Ontario COVID-19 vaccine certificate would also need to make accommodations for those vaccinated outside of Ontario and Canada. Currently, Ontario guidance considers individuals fully vaccinated at least two weeks after they have received a complete series of vaccines approved by Health Canada or those listed for emergency use by the World Health Organization. ${ }^{55}$

Globally, given that most vaccines have been delivered in high income countries, vaccine certificates will advantage individuals travelling from high income and vaccinerich countries and disadvantage those from countries without widespread vaccine access, or groups such as migrant workers and refugees. The economic and other consequences of such inequities ought to be minimized wherever feasible, including ensuring the vaccination of such individuals and groups in Canada.

Digital or app-based vaccine certificates may perpetuate inequalities in access to technology such as smartphones and internet access. Populations such as the economically disadvantaged, older adults, and those with disabilities may not have ready access or may find it more difficult to use such technologies. User-centric accommodations are important to support equitable access through various forms of vaccine certificates such as paper-based or voice-based, ${ }^{56}$ available by phone. Supports are important to assist those who cannot navigate the process of obtaining a vaccine certificate, and to communicate decisions about restricted access or enhanced precautionary measures in a fully accessible manner.

\section{Discrimination}

An additional equity consideration is how vaccine certificates will be regulated and enforced, and whether they could perpetuate disadvantage and prejudice. Black and Indigenous people experience over-policing and are more likely to be incarcerated in Canada..$^{57,58}$ As with COVID-19 precautions generally (e.g., lock-downs, curfews, physical distancing and masking requirements), there is a need to use strategies that will prevent disproportionate impacts on marginalized groups. Any enforcement of COVID-19 vaccine certificates must be calibrated to ensure that it does not contribute to further police harassment or state surveillance of marginalized populations, which in turn could lead to further vaccine hesitancy and mistrust. ${ }^{59}$

Vaccine certificates may also have an adverse effect on individuals with particular characteristics. Government and health authorities have an obligation to take 
into account the already disadvantaged positions of various groups. For example, differential impacts of vaccine certificates based on a protected ground such as disability (e.g., for those who for medical reasons cannot be vaccinated), ethnicity (e.g., minorities are more likely to be vaccine hesitant) or age (e.g., for those under the age of 12 who cannot yet access vaccination) must be considered and addressed. Procedural fairness will require meaningful engagement with populations who are most likely to be negatively impacted by vaccine certificates. Accommodation will need to be made for such groups wherever this is feasible, particularly in granting access to non-discretionary, public settings such as hospitals, LTC homes, schools, and universities. Greater efforts may be needed to address vaccine hesitancy in ways which are culturally appropriate and led by community leaders.

Vaccine certificates may be particularly important for people with disabilities defined as those with a physical, mental, intellectual, or sensory impairment. There is growing body of evidence indicating the disproportional impact of COVID-19 mortality and hospitalization for people with disabilities. ${ }^{60}$ It is possible that the continued absence of proof of vaccine requirements in high SARS-CoV-2 transmission settings may lead to greater social isolation and continued precautionary measures for people with disabilities, who are among the most susceptible to COVID-19.

\section{Privacy}

There are concerns that efficiency considerations, including the need to rapidly create a digital COVID-19 vaccine certificate, will trump privacy and data protection. ${ }^{14}$ Sharing personal health information with third party smartphone apps, and with individuals who are tasked with checking such apps with varying degrees of training, is an important concern. These technologies should undergo a privacy impact assessment and threat risk assessment which is made public except for any areas that would represent a security risk. Similarly, access to personal health information by private sector entities such as workplaces, entertainment and event venues remains controversial..$^{49}$ Importantly, the extent of personal health information contained on vaccine certificates should be limited to what is necessary to achieve the intended purpose, in accordance with the principle of least infringement. Privacy can be protected by digitalized, secure vaccine certificates with user-centric accommodations for those who need them.

\section{Charter Challenges and Human Rights Protections}

Some have raised concerns that requiring COVID-19 vaccine certificates to access public spaces such as schools or health care workplaces may be subject to challenges that this requirement infringes upon the Canadian Charter of Rights and Freedoms, ${ }^{61}$ such as the right to refuse medical treatment, including COVID-19 vaccination. In particular, section 7 of the Charter guarantees "life, liberty and security of the person"; section 15 protects against discrimination on protected grounds such as disability, age and ethnicity; and section 2(a) safeguards freedom of religion and conscience.

The Charter does not protect economic rights and consumer choices, such as air travel or work opportunities in high-risk discretionary settings if individuals choose not to be vaccinated. However, restricted access to non-discretionary public settings, such as schools and hospitals, may infringe upon Charter rights.

To withstand judicial scrutiny, any infringement upon Charter rights must be rationally connected to a compelling policy objective that is designed to minimally impair rights and accommodate marginalized groups and individuals who are likely to suffer a disproportionate impact. This includes alternative options for unvaccinated individuals to access and participate in activities and access settings requiring vaccine certification, including for example, the need to present a negative SARS-CoV-2 test result. Care must also be taken to ensure that adverse impacts of vaccination 
certificates are not disproportionate to the intended benefits. Safeguards including exemptions should be introduced to support individuals who cannot be vaccinated due to medical reasons to fully participate in society. Exemptions for both those who cannot be vaccinated for medical reasons, as well as those with philosophical or other reasons for declining COVID-19 vaccination should include access to essential public spaces including grocery stores and pharmacies. Importantly, the use of vaccine certificates should be subject to regular review and should only be required so long as it is justified by a compelling purpose. As COVID-19 vaccine coverage increases and the burden of SARS-CoV-2 decreases, the use of vaccination certificates may be more likely to be subject to Charter challenges.

\section{Guidance on Best Practices}

A report released by the Canadian Council of Parliamentary Ombudsman on May 26, 2021 developed guidance that provincial and territorial governments could use in establishing fairness principles for vaccine certificates. ${ }^{63}$ These include:

1. If vaccine certificates or passports are implemented in Canadian provinces and territories, governments must provide clear direction on their application and use to all entities providing services to the public, either via legislation or publicly available policy.

2. Government policy regarding the use of vaccination certificates or passports must be evidence informed and subject to regular review.

3. Determining access to public services based on vaccination status cannot be contrary to the pre-existing laws of the relevant jurisdiction.

4. If introduced, vaccine certification must be made available in a way that is equitable and accessible to everyone.

5. Requirements to disclose vaccination status in order to access public services must be proportionate to the type of service being provided, the associated risk to individuals and the risk posed to public health.

6. Accommodations must be made for those who have not received the vaccine.

7. Public organizations should provide clear guidance to their staff to assist them in making and communicating decisions to limit access to services based on vaccination status.

8. Decisions about restricting access to a service based on a person's vaccination status must be done in a transparent, procedurally fair manner and be clearly communicated to the affected person in an accessible way.

9. When decisions are made to deny or limit public services to those who may not be able to prove vaccination status, they must be informed of their right to appeal and be provided with information about the appeal process available.

10. If vaccine certificates or passports are implemented, government must ensure that independent oversight is in place.

\section{Interpretation}

COVID-19 vaccine certificates may have a practical short-term basis for supporting economic and societal reopening and a long-term basis as verifiable, secure, standardized, and portable electronic records of immunization. Some jurisdictions are also implementing vaccine certificates with the goal of incentivizing COVID-19 vaccination.

Given the strong scientific basis for vaccination reducing infection, hospitalization, and severe illness from COVID-19, limiting participation of higher-risk activities for 
SARS-CoV-2 transmission to vaccinated individuals who hold vaccine certificates should theoretically reduce the risk of transmission and infection to both vaccinated and non-vaccinated individuals.

Countries like Israel implemented COVID-19 vaccine certificates alongside reopening of higher transmission settings. While the Green Pass was in place, Israel did not see an increase in SARS-CoV-2 cases or COVID-19 hospitalizations and deaths.

Despite this, there is currently no direct scientific evidence of the impact of COVID-19 vaccine certificates on SARS-CoV-2 transmission or vaccine coverage. These should be areas of active research in countries like Israel which implemented COVID-19 vaccine certificates, and in those countries that did not. In the absence of these data, infectious diseases modelling could help estimate the potential benefit of vaccine certificates on SARS-CoV-2 transmission in higher-risk settings.

As of July $20,2021,79.6 \%$ of eligible Ontarians - those 12 years of age or older have received at least one dose of a COVID-19 vaccine and $62.2 \%$ are fully vaccinated with two doses. ${ }^{64}$ With Ontario having moved into phase 2 of its reopening plan on June 30, 2021 and already meeting the population vaccination targets for entry into phase 3 on July 16, 2021, there may currently be a very limited short-term role for using COVID-19 vaccine certificate to gain entry to higher SARS-CoV-2 transmission activities and settings. However, these reopening targets were established before the much more transmissible Delta variant become the dominant SARS-CoV-2 strain in Ontario, and other jurisdictions such as the United Kingdom and Israel have either had to delay reopening plans or reintroduce public health measures in the face of the Delta variant. Further, given the anticipated seasonality of SARS-CoV-2 as well as the future possibility of additional variants of concerns, establishing the infrastructure for COVID-19 vaccine certification may be useful.

Beyond their temporary potential use in accessing higher SARS-CoV-2 transmission settings and activities, COVID-19 vaccine certificates have several important potential long-term uses. This includes the ability to reliably verify the COVID-19 vaccination status of individuals as they return to schools and workplaces that require mandatory reporting of immunization status. In the absence of a national COVID-19 vaccine passports, there is also a need to verify vaccination status for returning Canadian travelers, and there are already emerging concerns about individuals intending to forge COVID-19 vaccination records to avoid quarantine requirements. ${ }^{65}$ Finally, as verifiable, secure, standardized, accessible, and portable records of immunization, COVID-19 vaccine certificates are likely to have individual and public health surveillance benefits.

There are important ethical, legal, accessibility and privacy considerations that should guide the design and implementation of COVID-19 vaccine certificates to ensure effects are not disproportionate to the aim. There are concerns that in the absence of government or health authority regulation, private sector entities will create their own requirements and systems. Provincial and territorial governments may want to consider development of a common design of vaccine certificates that reflects key development and implementation principles. Governments and health authorities can follow emerging international best practices to develop or regulate COVID-19 vaccine certificates with the necessary safeguards to protect privacy, human rights and enable accommodations. ${ }^{14}$

COVID-19 vaccine certificates may have a practical short-term utility for supporting and maintaining economic and societal reopening. In the longer-term, vaccine certificates may be useful as verifiable, secure, standardized, and accessible electronic or paper records of immunization. Further, given the anticipated seasonality of SARS-CoV-2 as well as the possibility of additional variants of concerns, establishing the infrastructure 
for COVID-19 vaccine certification may be useful if public health measures need to be reintroduced during future potential waves.

\section{Methods Used for This Science Brief}

We searched PubMed, Google Scholar, the COVID-19 Rapid Evidence Reviews, the Joanna Briggs Institute's COVID-19 Special Collection, LitCovid in PubMed, the Oxford COVID-19 Evidence Service, the World Health Organization's Global Literature on Coronavirus Disease, and other COVID-19 specific resources listed by the Guidelines International Network and the McMaster Health Forum. In addition, we retrieved reports citing relevant articles through Google Scholar and reviewed references from identified articles for additional studies. Jurisdictional information on COVID-19 vaccine certificates was obtained using relevant government and private sector websites. The search was last updated on June 11, 2021.

The COVID-19 Evidence Synthesis Network performed a research evidence scan for this Science Brief, published in an Evidence Synthesis Briefing Note. The COVID-19 Evidence Synthesis Network is comprised of organizations in Ontario's evidence synthesis and knowledge translation community who collectively provide high-quality, relevant, and timely synthesized research evidence about COVID-19. The Methods for the evidence scan can be found in the methods section of the Briefing Note. The evidence scan was last updated on June 8, 2021.

\section{Author Contributions}

GMK, KBB and NMS conceived the Science Brief. GMK, KBB, MDW and NMS wrote the first draft of the Science Brief. GMK performed the analyses. All authors revised the Science Brief critically for important intellectual content and approved the final version.

The authors would like to thank C. David Naylor for their comments on an earlier draft of this Science Brief.

\section{References}

1. Mithani SS, Bota B, Zhu DT, Wilson K. A scoping review of global vaccine certificate solutions for COVID-19. Res Sq. 2021;(2). https://doi.org/10.21203/rs.3.rs-334258/ v2

2. Phelan AL. COVID-19 immunity passports and vaccination certificates: scientific, equitable, and legal challenges. The Lancet. 2020;395(10237):1595-1598. https:// doi.org/10.1016/S0140-6736(20)31034-5

3. Voo TC, Reis AA, Thomé B, et al. Immunity certification for COVID-19: ethical considerations. Bull World Health Organ. 2021;99(2):155-161. https://doi. org/10.2471/BLT.20.280701

4. Chase $S$, Walsh M, Curry B. Travel rules to ease for fully vaccinated Canadians in July, while Ottawa works out COVID-19 vaccine passport standards. The Globe and Mail. https://www.theglobeandmail.com/politics/article-fully-vaccinatedcanadian-travellers-can-soon-return-home-without-14/. Published June 9, 2021. Accessed June 10, 2021.

5. Carbone S, Stalteri R, Plouffe R, et al. Key considerations and emerging evidence of experience across jurisdictions implementing documentation of vaccination. CanCOVID. Published online June 15, 2021:18. https://cancovid.ca/wp-content/ uploads/2021/07/CanCOVID-Issue-Note-Vaccination-Documentation-EN.pdf

6. Jonas S. Quebec may use vaccine passports this fall to shut out those not fully 
vaccinated from bars, gyms, festivals. CBC. Accessed July 13, 2021. https://www. cbc.ca/news/canada/montreal/digital-vaccine-passports-1.6094785

7. Research, Analysis, and Evaluation Branch. Evidence Synthesis Briefing Note: Guidance and Jurisdictional Evidence Regarding the Use of Vaccince Passports Evidence Synthesis Network. COVID-19 Evidence Synthesis Network; 2021. Accessed June 22, 2021. https://esnetwork.ca/briefings/guidance-and-jurisdictionalevidence-regarding-the-use-of-vaccince-passports/

8. Rosen K. What being fully vaccinated means for Manitoba restaurant patrons. CTV News. Published June 23, 2021. Accessed July 15, 2021. https://winnipeg. ctvnews.ca/what-being-fully-vaccinated-means-for-manitoba-restaurantpatrons-1.5482515

9. Government of Ontario. Reopening Ontario. Ontario.ca. Published April 27, 2020. Accessed June 10, 2021. https://www.ontario.ca/page/reopening-ontario

10. Simpson S. Majority of Canadians support vaccine passports for variety of indoor and outdoor activities. Ipsos. Published May 22, 2021. Accessed June 27, 2021. https://www.ipsos.com/en-ca/news-polls/majority-support-vaccine-passportsfor-variety-of-indoor-outdoor-activities

11. Vaccine Passports: Canadians show strong support for use in international travel, fewer willing to comply at home. Angus Reid Institute. Published May 26, 2021. Accessed June 10, 2021. https://angusreid.org/covid-vaccine-passport/

12. Interoperability of health certificates Trust framework. Published online March 12, 2021. Accessed June 22, 2021. https://ec.europa.eu/health/sites/default/ files/ehealth/docs/trust-framework_interoperability_certificates_en.pdf

13. Interim guidance for developing a Smart Vaccination Certificate. Published online March 19, 2021. Accessed June 10, 2021. https://www.who.int/publications/m/ item/interim-guidance-for-developing-a-smart-vaccination-certificate

14. Wilson K, Flood CM. Implementing digital passports for SARS-CoV-2 immunization in Canada. CMAJ. 2021;193(14):E486-E488. https://doi.org/10.1503/cmaj.210244

15. Government of Ontario. Vaccines and immunization. Ontario.ca. Published August 21, 2015. Accessed June 10, 2021. https://www.ontario.ca/page/vaccines

16. Katz GM, Born KB, Balicer RD, et al. Lessons learned from Israel's reopening during a nationwide COVID-19 vaccination campaign. Sci Briefs Ont COVID-19 Sci Advis Table. 2021;2(33). https://doi.org/10.47326/ocsat.2021.02.33.1.0

17. Thomas B, Flood CM, Krishnamurthy V, Tanner R, Wilson K. Vaccine ins and outs: an exploration of the legal issues raised by vaccine passports. $C D$ Howe Inst Work Pap. Published online July 13, 2021. Accessed July 13, 2021. https://www.cdhowe. org/public-policy-research/vaccine-ins-and-outs-exploration-legal-issues-raisedvaccine-passports

18. Ontario Agency for Health Protection and Promotion (Public Health Ontario). Focus on: backward contact tracing. Queens Print Ont. Published online May 2021:15. https://www. publichealthontario.ca/-/media/documents/ncov/phm/2021/05/ covid-19-backward-contact-tracing.pdf?la=en

19. Japan Ministry of Health, Labour and Welfare. Important notice for preventing COVID-19 outbreaks: avoid the "three Cs"! Ministry of Health, Labour and Welfare. Accessed June 22, 2021. https://www.mhlw.go.jp/content/10900000/000619576. pdf

20. Government of Canada. Coronavirus disease (COVID-19): prevention and risks. Canada.ca. Published September 14, 2020. Accessed June 22, 2021. https:// 
www.canada.ca/en/public-health/services/diseases/2019-novel-coronavirusinfection/prevention-risks.html

21. Kalvapalle R. $U$ of $T$ to require COVID-19 vaccinations for students living in residence. University of Toronto News. Published June 8, 2021. Accessed June 22, 2021. https://www.utoronto.ca/news/u-t-require-covid-19-vaccinationsstudents-living-residence

22. LeBel J. COVID-19: Vaccination required for Western University students in residence. Global News. Accessed June 22, 2021. https://globalnews.ca/ news/7898178/covid-19-western-university-mandatory-vaccination/

23. Bond M. Ryerson University latest school to make COVID-19 vaccination mandatory for students in residence - CityNews Toronto. CityNews. Published June 15, 2021. Accessed June 22, 2021. https://toronto.citynews.ca/2021/06/15/ ryerson-university-mandatory-covid-19-vaccination-in-residence/

24. Government of Ontario. Ontario mandates immunization policies for long-term care homes. Ontario.ca. Accessed June 22, 2021. https://news.ontario.ca/en/ release/1000230/ontario-mandates-immunization-policies-for-long-term-carehomes

25. Levitz S. COVID-19 border measures to ease in July for fully vaccinated Canadian travellers. thestar.com. Published June 21, 2021. Accessed June 22, 2021. https:// www.thestar.com/politics/federal/2021/06/21/covid-19-border-measures-toease-july-5-for-some-fully-vaccinated-travellers.html

26. Chase S, Walsh M. Fully vaccinated Canadians to be exempt from post-travel COVID-19 quarantine restrictions starting July 5. The Globe and Mail. Published June 21, 2021. Accessed June 22, 2021. https://www.theglobeandmail.com/ politics/article-fully-vaccinated-canadians-to-be-exempt-from-some-post-travelcovid-19/

27. Israel Ministry of Health. What is a Green Pass? Corona Traffic Light Model (Ramzor) Website. Accessed May 21, 2021. https://corona.health.gov.il/en/ directives/green-pass-info/

28. Israel Ministry of Health. Recovering from corona. GOV.IL. Published December 12, 2020. Accessed June 10, 2021. https://www.gov.il/he/departments/general/ corona-recovered-patients

29. EU Digital COVID Certificate. European Commission. Accessed June 10, 2021. https://ec.europa.eu/info/live-work-travel-eu/coronavirus-response/safe-covid19-vaccines-europeans/eu-digital-covid-certificate_en

30. Questions and answers - EU Digital COVID Certificate. European Commission. Published June 1, 2021. Accessed June 10, 2021. https://ec.europa.eu/ commission/presscorner/detail/en/QANDA_21_2781

31. Corona passport - where and how? corona | covid-19. Accessed June 22, 2021. http://en.coronasmitte.dk/corona-passport

32. Governor Cuomo announces launch of Excelsior Pass to help fast-track reopening of businesses and entertainment venues statewide. New York State: Governor Andrew M. Cuomo. Published March 26, 2021. Accessed June 10, 2021. https:// www.governor.ny.gov/news/governor-cuomo-announces-launch-excelsior-passhelp-fast-track-reopening-businesses-and

33. Excelsior Pass: frequently asked questions. New York State: COVID-19 Vaccine. Accessed June 10, 2021. https://covid19vaccine.health.ny.gov/excelsior-passfrequently-asked-questions 
34. Government of Israel. Second phase restrictions on reopening to take effect on Sunday, 21.2.2021. GOV.IL. Accessed May 14, 2021. https://www.gov.il/en/ Departments/news/19022021-03

35. Government of Israel. Government has approved the third reopening phase as planned, effective 7.3.2021. GOV.IL. Accessed May 14, 2021. https://www.gov.il/ en/departments/news/01032021-03

36. Government of Israel. Regulations for the Fourth Phase of the Reopening Program Approved, Effective 19.3.2021. GOV.IL. Accessed May 14, 2021. https://www.gov. il/en/Departments/news/18032021-03

37. Commission proposes update to coordinated travel measures ahead of the summer. European Commission. Published May 31, 2021. Accessed June 10, 2021. https://ec.europa.eu/commission/presscorner/detail/en/ip_21_2782

38. Dagan N, Barda N, Kepten E, et al. BNT162b2 mRNA Covid-19 Vaccine in a Nationwide Mass Vaccination Setting. N Engl J Med. 2021;384(15):1412-1423. https://doi.org/10.1056/NEJMoa2101765

39. McLaughlin K. France will require people to use a "health passport" to enter a restaurant or attraction this fall. Business Insider. Accessed July 13, 2021. https:// www.businessinsider.com/france-to-require-covid-19-vaccine-non-essentialactivities-2021-7

40. Statistiques défi vaccination Covid-19. Doctolib. Accessed July 13, 2021. https:// about.doctolib.fr/vaccination/statistiques.html

41. The Associated Press. France's Emmanuel Macron makes COVID-19 vaccination mandatory for all health workers. CBC. Published July 12, 2021. Accessed July 13, 2021. https://www.cbc.ca/news/world/france-covid-mandatory-vaccinationhealth-workers-1.6099589

42. Caulcutt C, Collis H. Macron's plan to beat back vaccine hesitancy. POLITICO. Published July 11, 2021. Accessed July 20, 2021. https://www.politico.eu/article/ france-covid-19-vaccination-vaccine-hesitancy-macron/

43. Hannah R, Ortiz-Ospina E, Beltekian D, et al. Coronavirus (COVID-19) Vaccinations. Our World in Data. Published January 21, 2021. Accessed January 21, 2021. https://ourworldindata.org/covid-vaccinations

44. CDC. When you've been fully vaccinated: how to protect yourself and others. Centers for Disease Control and Prevention. Published February 11, 2020. Accessed June 22, 2021. https://www.cdc.gov/coronavirus/2019-ncov/vaccines/ fully-vaccinated.html

45. Public Health Agency of Canada. Vaccinated against COVID-19? What does it mean to me? Canada.ca. Published June 25, 2021. Accessed July 1, 2021. https://www. canada.ca/en/public-health/services/diseases/2019-novel-coronavirus-infection/ awareness-resources/vaccinated-against-covid-19-public-health-measures.html

46. Wilf-Miron R, Myers V, Saban M. Incentivizing vaccination uptake: the "Green Pass" proposal in Israel. JAMA. 2021;325(15):1503. https://doi.org/10.1001/ jama.2021.4300

47. Tercatin R. Coronavirus: Israel launches 'Happy Badge' for weddings and large parties. The Jerusalem Post. Published July 16, 2021. Accessed July 16, 2021. https://www.jpost.com/breaking-news/coronavirus-in-israel-765-new-cases138-percent-of-tests-return-positive-673898

48. Phillips N. The coronavirus is here to stay - here's what that means. Nature. 2021;590(7846):382-384. https://doi.org/10.1038/d41586-021-00396-2 
49. Osama T, Razai MS, Majeed A. Covid-19 vaccine passports: access, equity, and ethics. BMJ. 2021;373:n861. https://doi.org/10.1136/bmj.n861

50. McKinnon B, Quach C, Dubé È, Nguyen CT, Zinszer K. Social and racial/ethnic differences in parental willingness to vaccinate children against COVID-19 in Montreal, Canada. medRxiv. Published online May 10, 2021:2021.05.08.21256831. https://doi.org/10.1101/2021.05.08.21256831

51. COVID-19 vaccine willingness among Canadian population groups. Statistics Canada. Published March 26, 2021. Accessed June 12, 2021. https://www150. statcan.gc.ca/n1/pub/45-28-0001/2021001/article/00011-eng.htm

52. Black scientists' task force on vaccine equity. City of Toronto. Published February 3, 2021. Accessed June 12, 2021. https://www.toronto.ca/news/black-scientiststask-force-on-vaccine-equity/

53. Barlow R. "Vaccine passports": COVID-19 protection or discrimination against BIPOC and the poor? Boston University. Published April 9, 2021. Accessed June 11, 2021. https://www.bu.edu/articles/2021/vaccine-passports-covid-19-protectionor-discrimination/

54. Brown KA, Stall NM, Joh E, et al. COVID-19 Vaccination Strategy for Ontario Using Age and Neighbourhood-Based Prioritization. Science Briefs of the Ontario COVID-19 Science Advisory Table; 2021. Accessed March 6, 2021. https://covid19sciencetable.ca/sciencebrief/covid-19-vaccination-strategy-for-ontario-usingage-and-neighbourhood-based-prioritization

55. COVID-19 guidance for individuals vaccinated outside of Ontario/Canada. Published online June 4, 2021. https://www.health.gov.on.ca/en/pro/programs/ publichealth/coronavirus/docs/vaccine/COVID-19_guidance_for_individuals_ vaccinated_outside_of_ontario.pdf

56. 12 challenges for vaccine passports. The Royal Society. Published February 19, 2021. Accessed June 10, 2021. https://royalsociety.org/news/2021/02/12challenges-for-vaccine-passports/

57. Morgan AN. Black Canadians and the justice system. Policy Options. Published May 8, 2018. Accessed June 11, 2021. https://policyoptions.irpp.org/magazines/ may-2018/black-canadians-justice-system/

58. Clark S. Overrepresentation of Indigenous people in the Canadian criminal justice system: causes and responses. Res Stat Div Dep Justice Can. Published online 2019:55. https://www.justice.gc.ca/eng/rp-pr/jr/oip-cjs/oip-cjs-en.pdf

59. Bajaj SS, Stanford FC. Beyond Tuskegee - vaccine distrust and everyday racism. $N$ Engl J Med. 2021;384(5):e12. https://doi.org/10.1056/NEJMpv2035827

60. Rotenberg S, Downer MB, Brown H, et al. COVID-19 vaccination for people with disabilities. Sci Briefs Ont COVID-19 Sci Advis Table. 2021;2(35). https://doi. org/10.47326/ocsat.2021.02.35.1.0

61. Thomas B, Flood CM. Eliminating religious and philosophical exemptions: the next step in Ontario's campaign against vaccine hesitancy. Healthc Policy Polit Sante. 2020;16(2):14-20. https://doi.org/10.12927/hcpol.2020.26357

62. Flood CM, Thomas B, Wilson $\mathrm{K}$. Mandatory vaccination for health care workers: an analysis of law and policy. CMAJ. 2021;193(6):E217-E220. https://doi.org/10.1503/ cmaj.202755

63. Fairness Principles for Public Service Providers Regarding the Use of COVID-19 Vaccine Certification. Canadian Council of Parliamentary Ombudsman; 2021:8. Accessed June 11, 2021. https://www.ombudsman.on.ca/Media/ombudsman/ 
ombudsman/resources/Brochures/CCPO-Fairness-Principles_Vaccine-PassportEN-May2021-accessible.pdf

64. Ontario vaccination data. COVID-19 Tracker Canada. Published July 20, 2021. Accessed July 20, 2021. https://covid19tracker.ca/provincevac.html?p=ON

65. Somos C. Federal agencies on alert for fake COVID-19 vaccine records. CTVNews. Published June 23, 2021. Accessed June 27, 2021. https://www.ctvnews.ca/ canada/federal-agencies-on-alert-for-fake-covid-19-vaccine-records-1.5482967 